\title{
Household Energy Consumption at an Open Market
}

\author{
D.M. Larruskain Eskobal ${ }^{1}$, O. Abarrategui Ranero ${ }^{1}$, E. Loroño Apraiz ${ }^{1}$, \\ M. D. Gutiérrez Ochoa de Alda ${ }^{1}$ and F. de la Bodega Bilbao ${ }^{1}$ \\ ${ }^{1}$ Department of Electrical Engineering \\ E.U.I.T.I., University of the Basque Country \\ Campus of Bizkaia -Plaza de la Casilla $\mathrm{n}^{\circ} 3,48012$ Bilbao (Spain) \\ phone:+34 946 014472, fax:+34 946014300 , \\ e-mail: ieplaesm@lg.ehu.es, \\ gauoihan@yahoo.es, ieploape@lg.ehu.es, iepguotm@lg.ehu.es, iepdebif@lg.ehu.es
}

\begin{abstract}
The European directive 2003/53/EC of 26 June 2003 opens the electricity market and settles down that the term limit for all the electricity customers to chose their providing company is $1^{\text {st }}$ July of 2007. Nevertheless, some Member States have gone beyond this term, Spain is among them, from the $1^{\text {st }}$ of January of 2003.
\end{abstract}

The customers and generators can freely make provision contracts, thus it is necessary to have a system that allows the consumption measurement and the energy transits between the different subjects and electrical activities.

The installation of new measuring equipment in the point of nondomestic qualified customers and network connection is compulsory, although it is not necessary to modify the meters for household customers. But if customers want it, they will be able to install an hour meter, since the consumed energy price has a different hour to hour price in the wholesale market.

Key words: energy liberalization, single-fase electricity meter, electronic control, low-cost.

\section{Introduction}

Mechanical electricity meters have been used in household electricity measurement for decades, but nowadays new, fully electronic meters are replacing mechanical meters. According with the new energy liberalization institutional framework, the future appears in the electronic energy meters.
Up to now, electronic meters have been limited by the analog-to-digital converter and the processor high prices.

In order to develop an electronic system, different microcontroller alternatives do exist, which allow a greater or smaller complexity based on the system needs.

\section{The Measurement Principle}

The principle used (Reduced Scan Principle) measures current and voltage in regular time intervals and multiplies the current and voltage samples. The multiplication results are summed up, with the sum representing the consumed energy (Ws, kWh). While the method normally used measures voltage and current at exactly the same time, the Reduced Scan Principle alternately measures voltage and current samples.

Every sample is used twice; once it is multiplied with the value measured before and once with the value measured afterwards. To further reduce the required multiplications, these two multiplications are reduced to one by using the sum of the two voltage samples. This measurement principle is shown in Fig. 2.

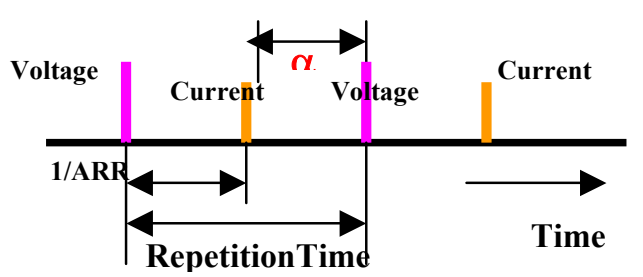

Fig. 1. Timing for the Reduced Scan Principle 
Where:

$\alpha$. Inherent Phase Shift of the Measurement Method [rad]

Repetition Time Length of a complete measurement cycle [s]

1/ARR Time Distance between two ADC Conversions [s]

The following shows the measurement sequence for a single-phase measurement. Current and voltage are measured alternately. The time, $\alpha$, represents the angle between related voltage and current samples.

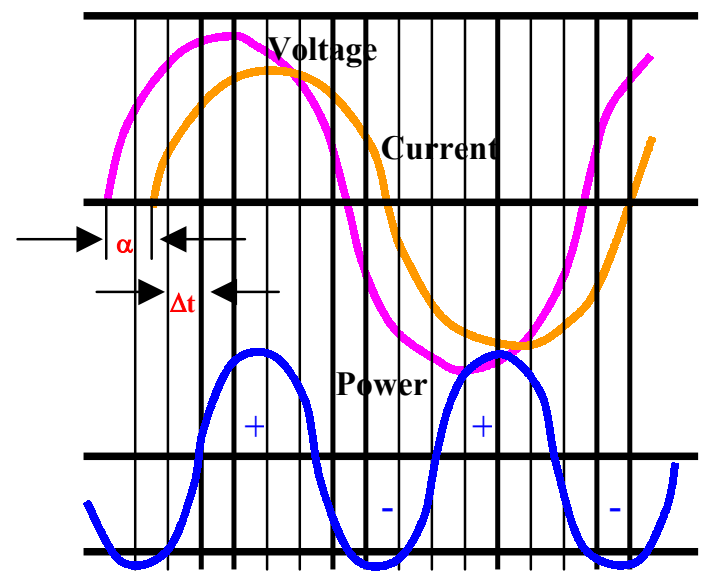

Fig. 2. Reduced Scan Measurement Principle

The measured energy $\mathrm{W}$ is:

$W=\sum_{t=0}^{t=\infty} i_{n} \times\left(u_{n-1}+u_{n+1}\right) \times \Delta t$

Where:

W Accumulated energy [Ws]

$i_{n} \quad$ Current sample at time $t_{n}[A]$

$u_{n-1}$ Voltage sample at time $t_{n-1}[\mathrm{~V}]$

$\mathrm{u}_{\mathrm{n}+1}$ Voltage sample at time $\mathrm{t}_{\mathrm{n}+1}[\mathrm{~V}]$

$\Delta \mathrm{t}$ Sampling interval between appertaining voltage and current measurements [s]

\subsection{The Inherent Error of the Reduced Scan Principle}

The Reduced Scan Principle has a small inherent error caused by the phase shift $\Delta \mathrm{t}$, once inductive and once capacitive, due to the time interval between voltage and current measurements. Any calculated energy sample shows this error, it is independent of the phase angle $\varphi$. between voltage and current. The value, e, of this error is:

$$
e=(\cos (\Delta t \times f \times 2 \pi)-1) \times 100
$$

Where:

e Error [\%]

$\Delta \mathrm{t}$ Sampling interval between voltage and current measurements [s]

f $\quad$ AC frequency $[\mathrm{Hz}]$

For example, with the values ( $\mathrm{f}=50 \mathrm{~Hz}, \Delta \mathrm{t}=$ $300 \mu \mathrm{s}$ ) the inherent error is $-0.444 \%$. This error can be eliminated during runtime by a

$$
c=\frac{1}{\cos (\Delta t \times f \times 2 \pi)}
$$

multiplication of the accumulated energy with the correction factor $\mathrm{c}$ :

The correction factor, $\mathrm{c}$, is normally included in the calibration constants (slope and offset) and

$$
F_{m d}=\left(\frac{\cos (\Delta t \times(f+\Delta f) \times 2 \pi)}{\cos (\Delta t \times f \times 2 \pi)}-1\right) \times 100
$$

not used explicitly.

\subsection{Measurement Error for Deviations of the AC Frequency}

If the ac frequency deviates from the nominal value used during the calibration, then a small error is generated. Table I shows this error dependent on the sample frequency and the ac frequency deviation. The introduced error, $F_{\mathrm{md}}$, is:

Table I. Errors dependent on the AC Frequency Deviation

\begin{tabular}{|c|c|c|c|c|}
\hline \multirow{2}{*}{$\begin{array}{c}\text { Measurement per } \\
\text { full Period }\end{array}$} & \multirow{2}{*}{$\begin{array}{c}\text { Sample Frequencies } \\
\text { Single Phase (50Hz) }\end{array}$} & \multicolumn{3}{|c|}{ Errors } \\
\cline { 3 - 5 } & & $\Delta \mathrm{f} / \mathrm{f}=+0.5 \%$ & $\Delta \mathrm{f} / \mathrm{f}=+1.0 \%$ & $\Delta \mathrm{f} / \mathrm{f}=+5.0 \%$ \\
\hline 20 & 1000 & $-0.051 \%$ & $-0.103 \%$ & $-0.523 \%$ \\
\hline 40 & 2000 & $-0.012 \%$ & $-0.025 \%$ & $-0.127 \%$ \\
\hline 80 & 4000 & $-0.003 \%$ & $-0.006 \%$ & $-0.030 \%$ \\
\hline 130 & 6500 & $-0.001 \%$ & $-0.002 \%$ & $-0.010 \%$ \\
\hline
\end{tabular}


Where:

$\mathrm{F}_{\mathrm{md}}$ Error due to the ac frequency deviation from the nominal frequency [\%]

$\Delta \mathrm{t}$ Time between related current and voltage samples [s]

f Nominal ac frequency (used during calibration) [Hz]

$\Delta \mathrm{f}$ Frequency deviation of the ac frequency during runtime $[\mathrm{Hz}]$

The errors for negative frequency deviations are the same as shown in Table I but with positive signs. The ADC is assumed to be error-free, this way only the influence of the frequency deviation is shown.

The additional error due to the deviation of the ac frequency can be reduced to nearly zero by the measurement of the actual ac frequency and an appropriate correction of the calculated energy.

\subsection{The Advantages of the Reduced Scan Principle}

- Only $50 \%$ of the measurements are necessary because every measured current or voltage sample is used twice

- Only $50 \%$ of the multiplications are necessary because two voltage samples are added before the multiplication

- Only one ADC is needed compared to up to six with the usual method.

- The computing power gained by reducing the number of multiplications can be used by the microcomputer for other system tasks.

- The Reduced Scan Principle is nearly independent of frequency deviations of the ac.

- The Reduced Scan Principle is also nearly independent of the interrupt latency time of the microcomputer.

\section{Single-Phase Electricity Meters}

The next two electronic electricity meter proposals are made for the measurement of European ac. From the utility, one phase and ground are wired into the house. In this way a nominal voltage of $230 \mathrm{~V}$ is available. The reduced scan principle is applied exactly as described in section 2 .
To measure the electric energy consumed, a current transformer or a shunt resistor is necessary, both solutions are shown. The voltage of the phase is also measured. With this configuration, the energy consumption of the load can be measured exactly. The measurement sequence for a single-phase meter is shown in Figure 1.

The ADC of the micro measures the voltage between the AVss and SVcc connections with a resolution of 14 bits. A split power supply with $+2.5 \mathrm{~V}$ and $-2.5 \mathrm{~V}$ is used. The common ground of the two power supplies has a voltage of onehalf of the voltage SVcc. This voltage is used as a base for the ADC voltages. The micro measures this base voltage at regular intervals and subtracts it from every measured current or voltage sample. In this way, signed measurement is possible.

To have a reference for the measurements a reference diode LM385-2.5 is used. The voltage of this diode is measured in regular intervals and the measured value is used as a base for the $\mathrm{SV} c$ relative $\mathrm{ADC}$ measurements.

\section{Current Measurement}

The main problem of the current measurement is the large dynamic range of the input values; ranging from $0.1 \%$ up to $1000 \%$ of the nominal value. The common methods used to solve this problem are shown in Figure 3 and are explained in the following text. If range switches are used, it is recommended that a hysteresis for the range selection criteria be used.

\subsection{Current Measurement With a Shunt}

The solution which uses a shunt resistor for the measurement of the load current is shown in Figure 4. The load current Iload flows through the shunt, which has a resistance of approximately $1.0 \mathrm{mOhm}$. The voltage drop at the shunt is amplified and measured by the micro.

A backup battery allows the time information (provided by the basic timer) to be kept and is also used during power-down periods. All current-consuming peripherals may be switched off. Therefore; the reference diode, the range switch, and the amplifier are switched off by the SVcc output. The EEPROM is switched off with 
a TP-output. A prepayment interface is connected to the micro. It allows the ac to be switched on after the insertion of a valid prepayment card.

\subsubsection{Shunt}

The load current IL flows through a resistor Rshunt $(0.3 \mathrm{mOhm}$, to $3.0 \mathrm{mOhm})$ and the voltage drop of this resistor (shunt) is used for the current measurement. Due to the small voltage drop, especially with low currents, it is necessary to amplify this voltage drop with an operational amplifier. This operational amplifier can have only a very small phase shift $\left(0.1 \_\right.$) to get the needed accuracy. The out- put voltage Vout, which is proportional to the current IL, is measured by the micro. The amount of Vout is:

$$
V_{\text {out }}=-I_{\text {load }} \times R_{\text {shunt }} \times \frac{R 2}{R 1}
$$

open switch, low current

$V_{\text {out }}=-I_{\text {load }} \times R_{\text {shunt }} \times \frac{R 2 / / R 3}{R 1}$

closed switch, high current

The value ki [A/step], used for the calculation of the meter constant $\mathrm{CZ}$ is:

$$
K_{i}=-\frac{S V_{C C}}{2^{14}} \times \frac{R 1}{R_{\text {shunt }} \times R 2}
$$

open switch, low current,

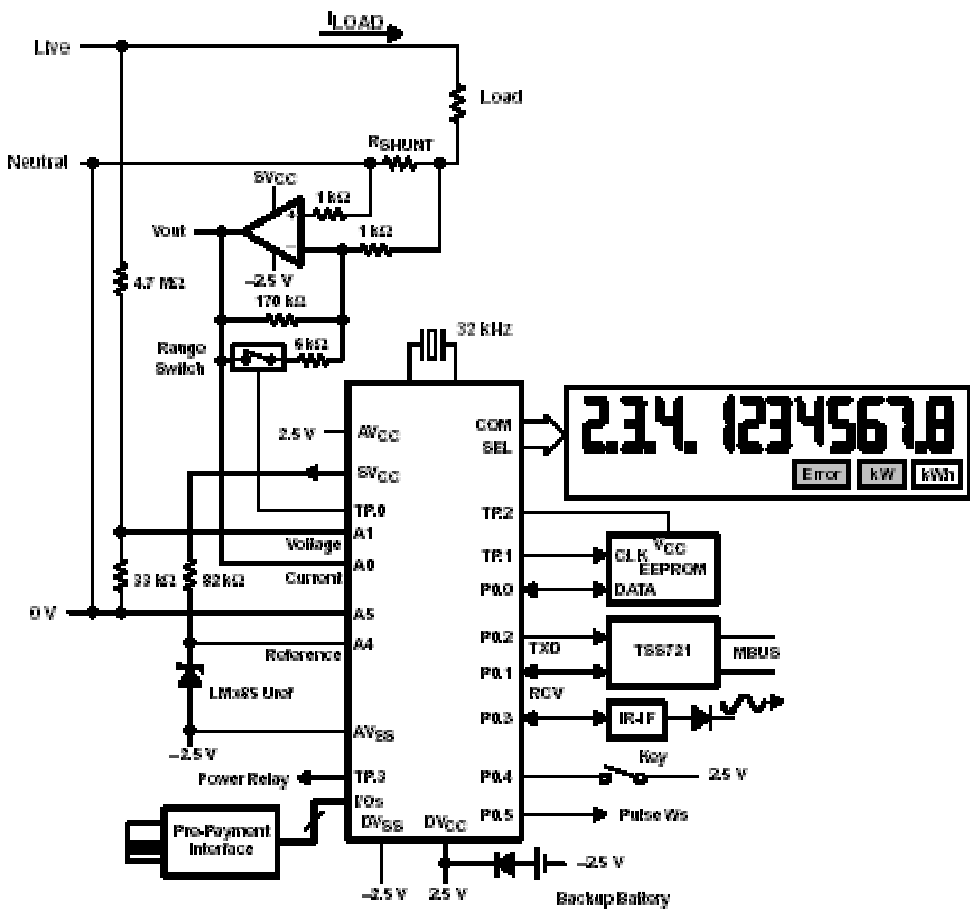

Figure 4. Single-Phase Electricity Meter With Shunt Resistor

$$
K_{i}=-\frac{S V_{C C}}{2^{14}} \times \frac{R 1}{R_{\text {shunt }} \times R 2 / / R 3}
$$

closed switch, high current

Advantages

- Resistive behavior

- Simple

- More than one range possible with switches

Disadvantages

- High losses with high currents

- Very low output voltage with small currents (amplifier necessary)

- Only usable with single-phase meters
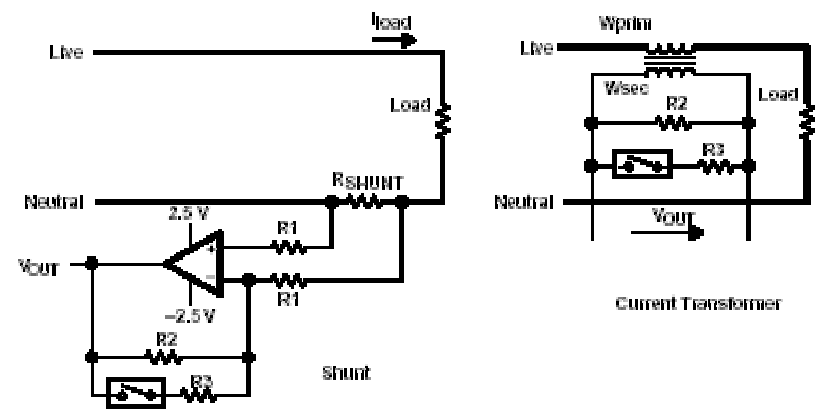

Current Tanssimer

Figure 3. Current Measurement 


\subsection{Current Measurement With a Current Transformer}

The solution, which uses a current transformer for the measurement of the load current, is shown in Figure 5. The secondary current Isec of the transformer flows through two paralleled resistors and generates a voltage Vsec which is measured by the micro. For currents greater than a certain value, the resistor with the lower value is switched on by the analog switch TLC4016. For low currents, this switch is opened to get a higher voltage and, therefore, a better resolution. The range switch algorithm uses a certain hysteresis to avoid too much switching.

If needed, additional current ranges can be implemented with the three analog switches of the TLC4016 that are not used. An AC Down signal out of the power supply connected to the interrupt $\mathrm{I} / \mathrm{O}$ terminal

P0.6 allows the micro to save important values (i.e., energy consumption) in the EEPROM in case of a power-fail.

The RF-readout module is connected to free outputs; this can be an unused segment line, a TP output, or an $\mathrm{I} / \mathrm{O}$ pin of Port0. The timing for the $\mathrm{RF}$ readout is made by the internal Basic Timer. It delivers the needed interrupt frequencies. The supply voltage needed for the RF interface is done with a step-up voltage supply. It transforms the available $5 \mathrm{~V}$ to $6 \mathrm{~V}$ or more.

\subsubsection{Transformer}

The secondary current Isec of the current transformer, which is

$$
I_{\text {sec }}=\frac{W_{\text {prim }}}{W_{\text {sec }}} \times I_{\text {load }}
$$

flows through a resistance Rsec (the resulting resistance of the two resistors $\mathrm{R} 2$ and $\mathrm{R} 3$ ) and generates a voltage VOUT, which is measured by the micro:

$$
V_{\text {out }}=\frac{W_{\text {prim }}}{W_{\mathrm{sec}}} \times I_{\text {load }} \times R_{\mathrm{sec}}
$$

Where:

$\mathrm{Rsec}=\mathrm{R} 2 \quad$ (switch open, low currents) $\mathrm{Rsec}=\mathrm{R} 2 \| \mathrm{R} 3 \quad$ (switch closed, high currents) The value ki [A/step], used for the calculation of the meter constant $\mathrm{CZ}$

$$
K_{i}=-\frac{S V_{C C}}{2^{14}} \times \frac{W_{\mathrm{sec}}}{R_{\mathrm{sec}} \times W_{\text {prim }}}
$$

Advantages

- Isolation from ac

- High accuracy for the magnitude of the current ( $0.1 \%$ reachable)

- More than one range possible with switched resistor.

Disadvantages

- Sensible to de current: may lead to saturation

- Costly

\section{Measurement of Voltage, Current,} Apparent Power, and Reactive Power

The reduced scan principle measures only active power. If reactive power or apparent power is to be measured, other methods have to be used. The implemented measurement method also depends on the main application of the electricity meter.

A meter for the measurement of reactive power only probably uses a different algorithm than an electricity meter for active power that does he reactive power measurement as a background task only. This section shows simple methods that use as much as possible the voltage and current samples measured for the active power calculation.

\subsection{Measurement of Voltage and Current}

The measurement of voltage and current is possible by summing up the absolute values of the ADC results during integer numbers of full periods. The result is an indication of the average value of the voltage Vavrg respective of the current Iavrg. If corrected as shown, the current and voltage values can be used for other purposes.

The formula for a sinusoidal voltage is shown in the following. The one for the current is equivalent to it.

$$
\begin{gathered}
K_{i}=\frac{V_{\text {peak }} \times 2}{p} \\
V_{\text {eff }}=\frac{V_{\text {peak }}}{\sqrt{2}} \rightarrow V_{\text {eff }}=\frac{V_{\text {avrg }} \times p}{2 \sqrt{2}} \cong 1.11 \times V_{\text {avrg }}
\end{gathered}
$$




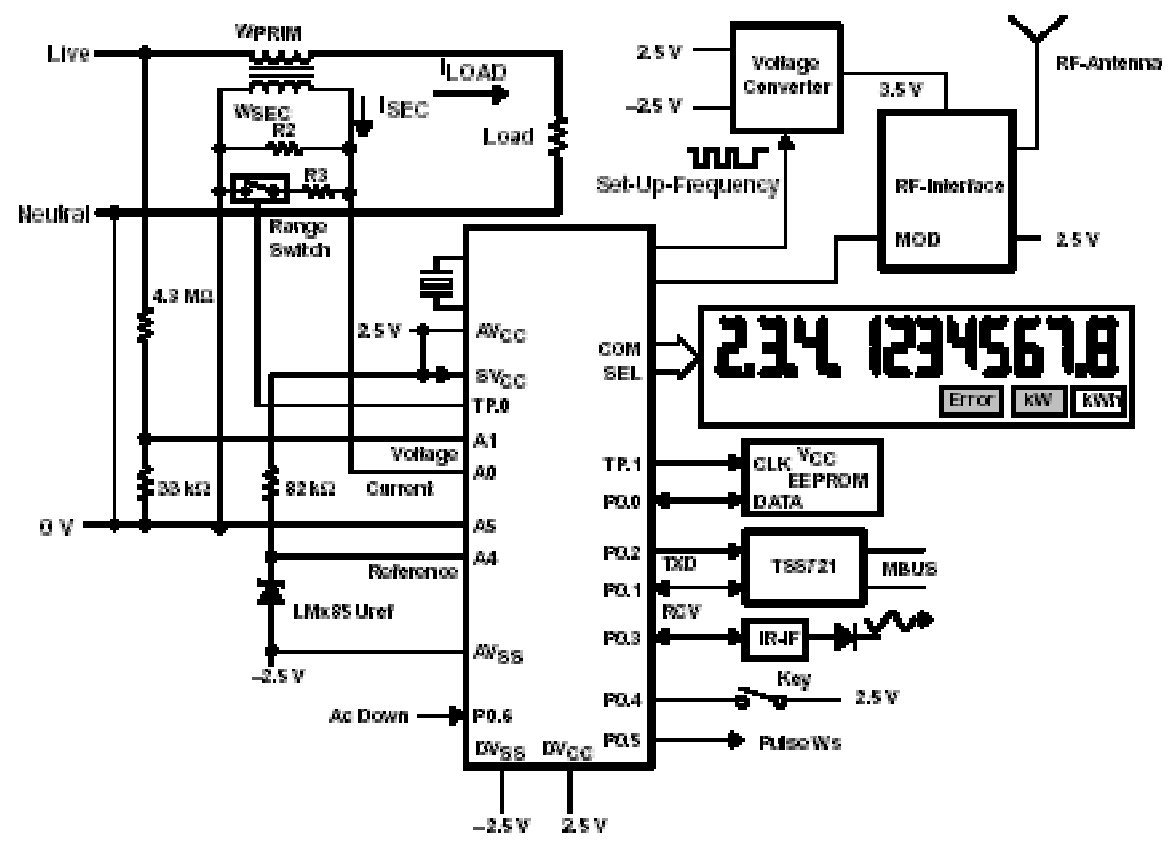

Figure 5. Single-Phase Electricity Meter With Current Transformer and RF Readout

\subsection{Measurement of the Apparent Power}

The apparent power is defined by the formula Papp $=\mathrm{U} \times \mathrm{I}$. There is no exact definition for the apparent power when harmonics are included. A possible solution is to use the voltage and current samples taken for the active power measurement. These samples are made absolute and summed up for an integer number of ac periods. If these summed-up values, representing the average value, are multiplied and corrected the apparent power is the result.

The correction is necessary due to the difference of the average value and the effective value of a sinusoidal current or voltage. The apparent energy Wapp is:

$$
W_{\text {app }}=V_{\text {avrg }} \times I_{\text {avrg }} \times 1.11^{2} \times t
$$

\subsection{Measurement of the Reactive Power}

Two simple methods exist for the measurement of the reactive power:

- Delay of the voltage (or current) samples for the time representing $90^{\circ}(\pi / 2)$ of the ac frequency.
- Calculation of the apparent power and the active power

\subsubsection{Delay of Samples}

With a carefully chosen sampling frequency, the angle $90^{\circ}(\pi / 2)$ can be made an integer multiple of the sampling interval. If each voltage sample is delayed with a RAM-based by this integer number and multiplied with the actual current sample, the result is the reactive power.

EXAMPLE: ac frequency $50 \mathrm{~Hz}, 90^{\circ}$ are $5 \mathrm{~ms}$, with a sampling frequency of $2000 \mathrm{~Hz}$ the necessary FIFO buffer is $5 \mathrm{~ms} \times 2000 \mathrm{~Hz}=10$ words. For every phase 20 bytes of RAM are needed for the FIFO.

\subsubsection{Calculation out of the Apparent Power}

The apparent power is calculated as described in Section 3.2. The reactive power is calculated with the values of the active power and the apparent power by the formula:

$$
W_{\text {react }}=\sqrt{W_{a p p}^{2}-W_{a c t}^{2}}
$$




\section{Electronic system functionality}

\subsection{The Analog-to-Digital Converter of the micro}

The analog-to-digital converter (ADC) of the micro measures the voltage between its AVss and SVcc connections with a resolution of 14 bits. The signed voltages coming from the current and voltage interfaces are shifted into the unsigned range of the ADC by simple interfaces described below. The micro subtracts the measured or calculated offset value from every measured current or voltage sample: this enables signed, offset corrected measurements.

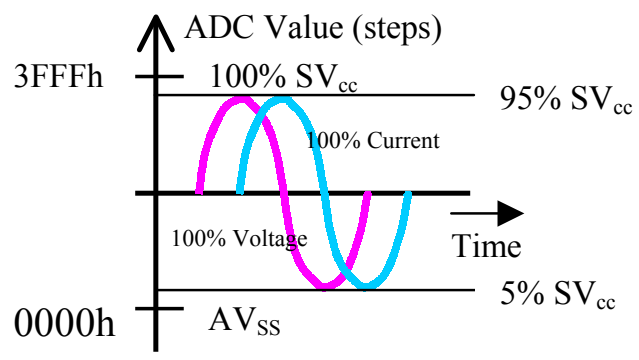

Figure 6. Allocation of the ADC Range

Figure 6 shows the placement of the current and voltage coming from the voltage dividers and the current interfaces into the analog-to-digital converter's range. All calculations and proposals base on a use of $90 \%$ of the ADC range for nominal $(100 \%)$ values of current and voltage. This means up to $111 \%$ of the nominal values are still measured correctly. This allocation may be changed if necessary.

Table II shows the influence of the analog-todigital converter's performance to the accuracy of the measurement of the electric energy. Two influences are involved:

1) The deviation of the $\mathrm{ADC}$ from the linearity. Each one of the four ranges A, $\mathrm{B}, \mathrm{C}$ and $\mathrm{D}$ has calculated deviations up to 20 ADC steps compared to the two ranges bordering on it.

2) The saturation effect at the range limits: if the sample for the definition of the range is taken in another range than the sample for the 12-bit conversion (36 ADCLKs later) than the result is $\mathrm{xFFFh}$ for increasing input signals and $\mathrm{x} 000 \mathrm{~h}$ for decreasing input signals $(\mathrm{x}$ denotes the number of the range where the range sample was taken). As the results show, the two saturation effects compensate nearly to zero.

The deviations of the analog-to-digital converter used with the examples below ( $+/-20$ steps) are greater than the specified ones. These large deviations are used only to show the relative independence of the overall accuracy from the ADC error. The actual, specified deviations are $+/-10$ steps. It is recommended not to use the exact midpoint of the supply voltage $\mathrm{Vcc}(\mathrm{Vcc} / 2)$ for the common reference point. This is due to the possible slight slope deviation at the border of two ADC ranges (here $\mathrm{B}$ and $\mathrm{C}$ ). This may influence the accuracy for the lowest currents. Table II shows also the influence for some extreme deviations of the analog to-digital converter characteristic. Figure 6 explains the meaning of the used graphics: it shows the second deviation curve of Table II in detail.

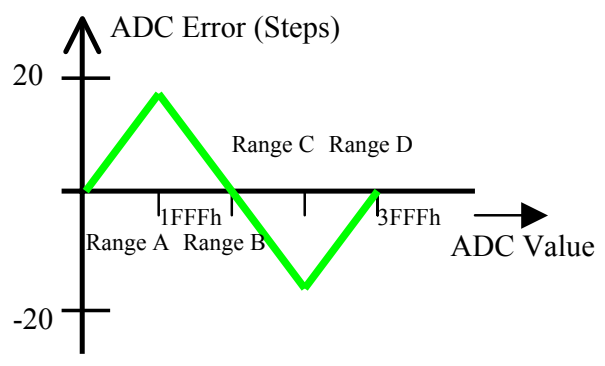

Figure 7. Explanation of ADC Deviation (2nd Column of Table II)

The function shows the deviation at any point of the four ADC ranges. Due to the monotony of the ADC the errors at the range limits are always equal. The errors shown in Table II were calculated with a software program. The following steps were taken:

1) Measurement and calculation of the error at $5 \%$ of the nominal current.

2) Measurement and calculation of the error at $100 \%$ of the nominal current

3) Calculation of the slope and offset for the correction (calibration)

4) Simulation of voltage and current samples: any sample is modified with the ADC error (exactly like during calibration).

5) Correction of all measured values with the calculated slope and offset 
6) Calculation of the resulting error

The saturation effect at the range limits is always included. The first column of Table II with an ideal ADC characteristic (zero deviation) shows only this effect and the finite ADC resolution. This column can be used as a reference for the errors of the other five columns.

The calculations are made with the following conditions:

- Virtual Ground location in the ADC range: 8190 steps (1FFEh) $49.98 \%$ of full ADC range

- Measurement time for calibration points: $5 \mathrm{~s}$ (calibration points are measured this time)

- Measurement time for different loads: 9s

- AC frequency: $50 \mathrm{~Hz}$

- Cosine $\varphi: 1\left(0^{\circ}\right)$
- Sample frequency: $2048 \mathrm{~Hz}$ (488.3 $\mu \mathrm{s}$ sample distance)

- Voltage: 100\% Vpp uses $90 \%$ of the ADC range

- Current: $100 \%$ Ipp uses $90 \%$ of the ADC range

The drawings on top of the columns of Table II indicate the ADC error in dependence of the ADC value. Figure 7 shows the drawing above the second column in a magnified form.

The large errors at $0.1 \%$ of the nominal current result from the relatively far distance from the $5 \%$ calibration point and from the missing resolution of the ADC at this small load. The peak-to-peak value of the ADC result is only 14.7 steps. These errors can be reduced drastically by using one of the following methods.

Table II. Errors With One Current Range and Single Calibration Range

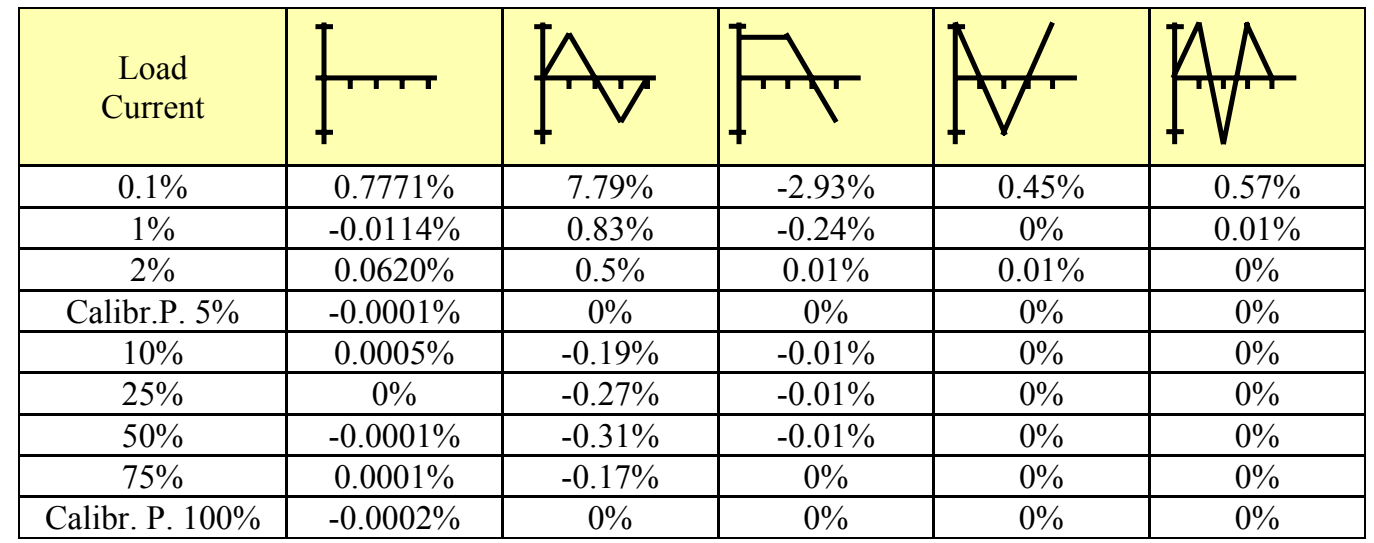

\section{Conclusion}

The electronic electricity meter proposal is made for the measurement of European ac for household users. The low-cost electricity meter measures voltage, current, apparent power and reactive power with high accuracy and keeps an historical consumption registry storage, which allows a statistical analysis of the consumption. The reading can be done via modem, avoiding displacements.

\section{References}

[1] Directive 2003/54/EC of the European Parliament and of the Council of 26 June 2003 concerning common rules for the internal market in electricity and repealing Directive 96/92/EC

[2] Ley 54/1997 Ley del sector eléctrico

[3] RD 2018/1997 Reglamento de puntos de medida

[4] Rudolf F. Graf, "Circuitos de medida”, Ed. Paraninf 1996

[5] MSP 430 Family, "Metering application report", literature number SLAUE10C

[6] MSP 430 Family, "Application Report", literature number SLAA024 